\title{
LOS TEMPORALES DE LLUVIA Y VIENTO EN GALICIA. PROPUESTA DE CLASIFICACIÓN Y ANÁLISIS DE TENDENCIAS (1961-2001)
}

\author{
Manuel Cabalar Fuentes \\ Departamento de Xeografía \\ Universidade de Santiago de Compostela
}

\section{RESUMEN}

En el presente artículo se intenta definir una propuesta de clasificación de los episodios de temporal de lluvia y viento en Galicia atendiendo a su intensidad y potencial peligrosidad, en la que se tendrá en cuenta la combinación de tres factores: precipitación media diaria del episodio, valor medio de las rachas máximas de viento y duración del evento. Asimismo, se analiza la evolución cronológica de los episodios de temporal en los últimos 40 años (período 1961-2001).

Palabras clave: Temporal de lluvia y viento, intensidad, peligrosidad, Galicia.

\section{RÉSUMÉ}

Dans ce travail on prétende definir une classification des épisodes de têmpete de pluie et vent en Galice, selon leur intensité et leur dangereux; nous avons combiné trois facteurs: volume moyen de pluie du episode, valeur moyen des rafales maximals du vent et duration du episode. On analyse aussi l'évolution cronologique des épisodes de têmpete dans les derniers 40 ans (1961-2001).

Mots clés: têmpete de pluie et vent, intensité, peligrosité, Galice.

\section{ABSTRACT}

In this paper we try to define a classification of storms of rain and wind in Galicia with regard to their intensity and potential dangerousness, bearing in mind the combination of three factors: daily average precipitation in the episode, average value of the greatest gusts of wind, and duration of the event. Also, we focus on the chronological evolution of these events the latest 40 years (period: 1961-2001).

Key words: rain and wind storms, intensity, dangerousness, Galicia. 


\section{Introducción}

Por su ubicación en la fachada atlántica del continente europeo, Galicia es un territorio estratégicamente situado en el camino de los centros de bajas presiones del Frente Polar que se generan en el Atlántico Norte. Estos centros depresionarios alcanzan el litoral europeo animadas por el sistema de circulación de vientos del W que domina en estas latitudes, muy en particular durante el semestre invernal del año (Octubre-Marzo), momento en el que estas bajas presiones alcanzan su máxima fuerza por la mayor diferencia de temperatura entre la cubeta polar y el trópico. Es entonces cuando el cinturón planetario de vientos de poniente alcanza su máxima expansión hacia el S en el Hemisferio Boreal.

Es en estos meses cuando Galicia se ve directamente afectada por el frecuente paso de estas perturbaciones oceánicas, que en numerosas ocasiones traen consigo fuertes rachas de viento y precipitaciones de cierta intensidad, lo que en la terminología climática se denominan como temporales de lluvia y viento. Tales condiciones meteorológicas provocan muy a menudo importantes problemas socioeconómicos, con serio trastorno de las actividades cotidianas de la población y daños en las infraestructuras, en especial en la orla litoral y proximidades. De este modo, las inundaciones de inmuebles y campos de cultivo, los prolongados cortes de fluído eléctrico y de las líneas telefónicas, los daños en edificios por las rachas de viento, el amarre a puerto de las flotas pesqueras y mercantes e incluso los naufragios, acostumbran ser noticia casi todos los inviernos en nuestra región.

Pero a pesar de constituir un riesgo climático de primer orden, en la bibliografía especializada tanto española como gallega son muy contados los trabajos sobre esta temática. A nivel nacional, sólo podemos resaltar las recientes aportaciones de RASILLA et alt (2002) sobre los temporales de viento en Cantabria, así como la obra de síntesis sobre los riesgos climáticos en la Península de OLCINA (1994) en la que, sin embargo, son tratados por separado los temporales de lluvia y los de viento. Y en el ámbito gallego encontramos diversas aportaciones centradas, tanto en el aspecto sinóptico de estos eventos (CASTILLO y PÉREZ, 1993; CASTILLO, 2001), como en el tipo de daños ocasionados, recurrencia y zonificación de espacios más afectados (MARTÍ, GARCÍA y MIRAGAYA, 1998; MARTÍ y PÉREZ SÁNCHEZ 1997; MARTÍ, 2002). Se carece, por tanto, de un análisis concreto sobre la frecuencia de los episodios de temporal, así como de un estudio de sus aspectos cualitativos y cuantitativos, e incluso de su evolución cronológica, es decir, el hecho de averiguar si con el paso de los años existe una tendencia de aumento o disminución de su recurrencia e intensidad.

Pretendemos, pues, rellenar en lo posible un vacío muy significativo en los estudios de climatología de Galicia, siquiera sea parcialmente. Así pues, abordaremos el estudio de los episodios de temporal de lluvia y viento desde una óptica fundamentalmente cualitativa, proponiendo una clasificación de los mismos referida a su intensidad y su grado estimado de peligrosidad; y también con un enfoque cronológico, intentando discernir si en los últimos 40 períodos invernales los temporales han modificado su frecuencia o intensidad.

\section{Metodología}

Para llevar a cabo este análisis y clasificación de los temporales, tomamos como base de partida los datos diarios de precipitación y racha máxima de viento de 3 observatorios principales de la red del INM que cubren de $\mathrm{N}$ a S la fachada atlántica gallega: A Coruña «Instituto», Santiago «Lavacolla», y Vigo «Peinador» (ver Figura 1), a los que previamente se 
les han aplicado los correspondientes tests de homogeneidad para verificar su fiabilidad (test de las rachas y de Hellmert) con resultados satisfactorios. Estos datos pertenecen exclusivamente a los semestres del año que coinciden con el momento en el que estos eventos son más frecuentes (desde el mes de Octubre hasta el de Marzo, ambos inclusive), en el período de tiempo comprendido entre el invierno 1961-62 (es decir, desde Octubre de 1961 hasta Marzo de 1962) y el invierno 2000-2001, que en muchos puntos de Galicia será recordado por haber batido récords de pluviosidad desde que existen los registros regulares de datos (MARTí, 2002). Así pues, abarca un total de 40 años, de entrada un período de tiempo representativo para poder sacar conclusiones de cierta fiabilidad. Conviene aclarar que para el caso del observatorio de A Coruña, no pudimos conseguir los datos diarios referentes a las rachas máximas de viento para el período comprendido entre Diciembre de 1971 y Marzo de 1974, hecho que nos ha imposibilitado localizar episodios de temporal en dicho observatorio durante ese período de tiempo.

A partir de estos datos, se seleccionaron en cada uno de los observatorios los episodios de temporal de lluvia y viento que iban apareciendo. Era pues necesario fijar un determinado criterio para discriminar los temporales de lluvia y viento, y la verdad es que el hecho de establecerlo fue una labor ciertamente compleja ante la falta total de referencias y precedentes al respecto. Esto implicaba seleccionar por nosotros mismos, y siguiendo unos criterios lo más objetivos posible, unos determinados umbrales mínimos de precipitación y viento com-

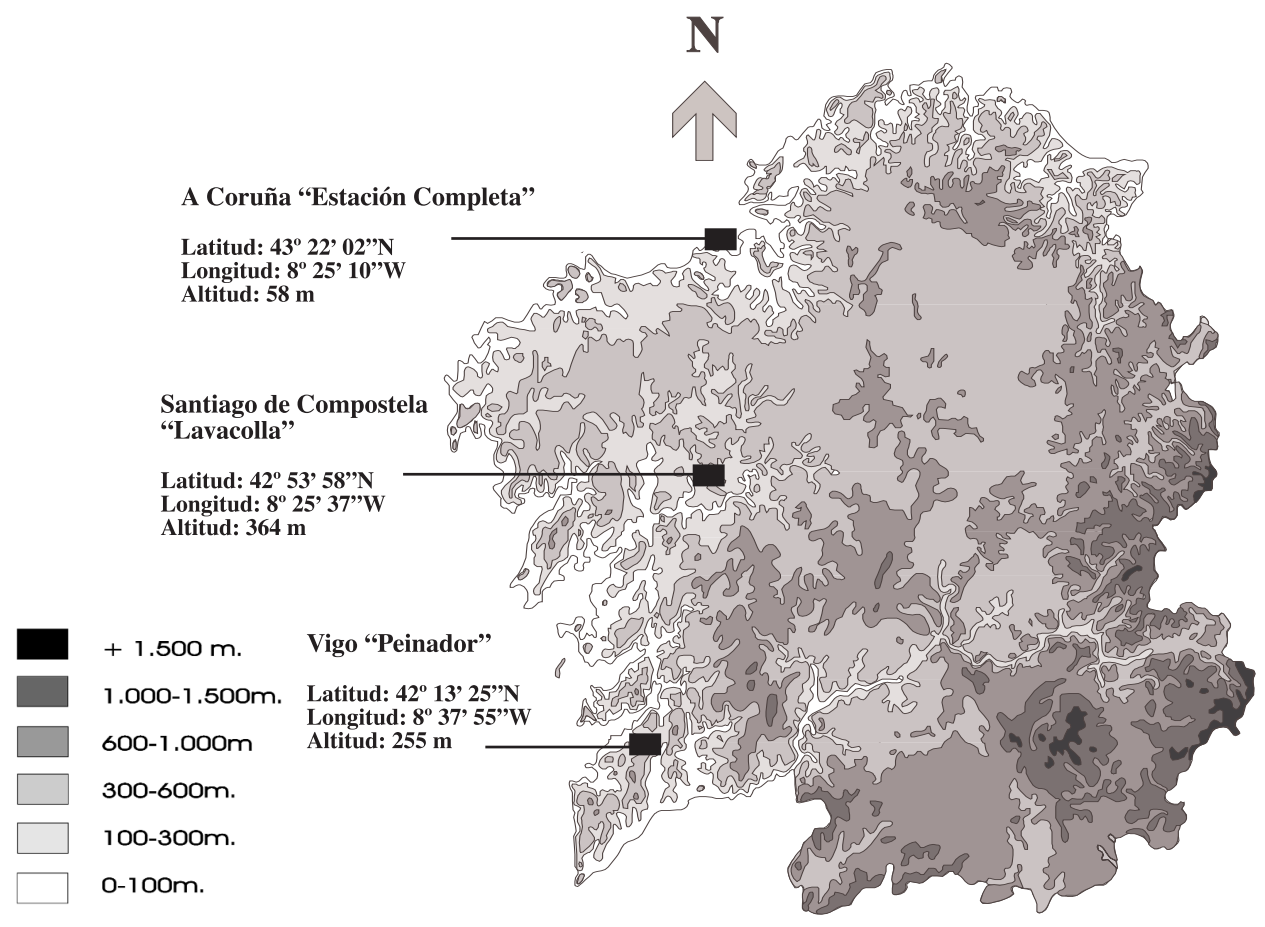

Fuente: Coordenadas de localización procedentes del INM. Elaboración propia.

FIGURA 1. Localización de los observatorios. 
binándolos adecuadamente. $\mathrm{Al}$ fin optamos por el siguiente método: consideramos episodio de temporal toda aquella serie de días consecutivos que registran una precipitación superior a $30 \mathrm{~mm}$ (umbral más empleado para distinguir las precipitaciones fuertes) y/o una racha máxima de viento igual o superior a $62 \mathrm{Km} / \mathrm{h}$ (umbral mínimo de la escala de Beaufort para la consideración de situación de temporal de viento). Se trata de que ambos umbrales se alcancen en días consecutivos aunque sea de forma alterna (por ejemplo: que en una serie de 5 días consecutivos los dos primeros registren una racha de viento igual o superior a 62 $\mathrm{Km} / \mathrm{h}$, y los tres siguientes superen los $30 \mathrm{~mm}$ de precipitación). Y la razón es bastante simple; habitualmente no coinciden el mismo día las rachas de viento más fuertes y las mayores precipitaciones, sino que unas suelen preceder a las otras según se desarrolle la situación atmosférica en cuestión, y sin que por eso dejemos de estar ante una misma situación de temporal (pautas de tránsito de los sistemas frontales: vientos racheados frecuentemente preceden a la llegada de los frentes nubosos que aportan las precipitaciones). En el caso de que ambas características sí coincidan el mismo día, hablaríamos de un día de temporal, que necesariamente forma parte de un episodio de temporal, pero no tiene por qué estar siempre presente en dichos eventos. El episodio en cuestión se da por concluído cuando encontramos dos o más días consecutivos donde no se cumple ninguna de las dos condiciones antes señaladas. Es absolutamente necesario que las dos manifestaciones atmosféricas extremas tengan lugar; así, en el caso hipotético de que durante una serie de días consecutivos las precipitaciones superaran los $30 \mathrm{~mm}$ pero las rachas máximas de viento no llegaran a sobrepasar el umbral de los $62 \mathrm{Km} / \mathrm{h}$, o viceversa, no se podría hablar con propiedad de un temporal de lluvia y viento, sino de un temporal de lluvia o de viento según el caso.

Una vez definidos y localizados los episodios de temporal, el siguiente paso necesario ha sido clasificarlos según su intensidad y grado estimado de peligrosidad, materia central del presente trabajo. Esta misión no fue nada sencilla, pues también se carece de precedentes de clasificaciones de este tipo en nuestro ámbito de investigación más próximo. Hubo pues que establecer una clasificación basada en criterios propios, y finalmente optamos por combinar el valor medio diario de la precipitación recogida durante cada episodio y la velocidad media diaria de las rachas máximas de viento, entendiendo que éste era mejor criterio para valorar la intensidad que escoger valores extremos, que como tales casi siempre son puntuales (la existencia de elevados valores medios tanto puede implicar valores extremos muy acusados como valores que se mantienen elevados de un modo sostenido). Así pues, para el valor medio de las rachas máximas de viento se escogieron los diferentes umbrales establecidos en la escala de Beaufort para medir la fuerza del viento, de tal suerte que las medias inferiores a $62 \mathrm{Km} / \mathrm{h}$ (umbral mínimo de las rachas atemporaladas en esta escala) serán consideradas débiles, mientras que las medias superiores a $88 \mathrm{Km} / \mathrm{h}$ (umbral que distingue las rachas muy intensas) serán etiquetadas como intensas.

Para las precipitaciones decidimos tomar los valores medios diarios de los episodios de temporal de los 3 observatorios estudiados y calcular los quintiles resultantes (el quintil es el punto de referencia aconsejado por la OMM para el estudio de elevadas pluviosidades), siendo los valores inferiores al $1^{\circ}$ quintil los considerados débiles y los superiores al $5^{\circ}$ quintil los intensos. Finalmente era necesario combinar ambos factores para establecer la intensidad efectiva de los temporales, y a tal fin elaboramos una matriz en la que cruzamos los dos valores: así, por poner los dos ejemplos extremos, los episodios de temporal con una precipitación media diaria inferior al $1^{\circ}$ quintil y una media de las rachas máximas de viento inferior a $62 \mathrm{Km} / \mathrm{h}$ serían clasificados como débiles, mientras que los que tuviesen una precipitación media diaria superior al $5^{\circ}$ quintil y una media de las rachas máximas de viento superior a $88 \mathrm{Km} / \mathrm{h}$ serían considerados como intensos. El resultado de la matriz clasificadora se puede observar con detalle en el CUADRO 1. 
Cuadro 1.

INTENSIDAD DE LOS TEMPORALES DE LLUVIA Y VIENTO

Precipitación media diaria

\begin{tabular}{|l|c|c|c|c|}
\hline Rachas máximas & $<\mathbf{2 1} \mathbf{~ m m}$ & $\mathbf{2 1 - 3 0} \mathbf{~ m m}$ & $\mathbf{3 0 - 4 2} \mathbf{~ m m}$ & $>\mathbf{4 2} \mathbf{~ m m}$ \\
\hline $30-61 \mathrm{Km} / \mathrm{h}$ & Débil & Débil & Moderado & Fuerte \\
\hline $62-74 \mathrm{Km} / \mathrm{h}$ & Débil & Moderado & Moderado & Fuerte \\
\hline $75-88 \mathrm{Km} / \mathrm{h}$ & Moderado & Moderado & Fuerte & Intenso \\
\hline$=>89 \mathrm{Km} / \mathrm{h}$ & Fuerte & Fuerte & Intenso & Intenso \\
\hline
\end{tabular}

Elaboración propia.

Pero también se ha tenido en cuenta un hecho tan importante de estos eventos climáticos como su duración (persistencia), pues como es lógico su potencial capacidad para generar daños se hace mayor cuanto más se prolongue en el tiempo. Con esto abordamos la clasificación de los temporales en función de su grado estimado de peligrosidad, teniendo muy presente como ya dijimos antes el hecho de que al margen de lo intenso que sea un temporal, cuanto más dure peores serán sus consecuencias. Por lo tanto, a los criterios de intensidad antes establecidos se le añade la duración de los episodios. Con esta nueva variable en juego, se elabora una nueva matriz donde cruzamos los grados de intensidad anteriormente establecidos con ciertos intervalos de duración de los episodios que nosotros mismos hemos escogido considerándolos como los más coherentes: de 1 a 2 días como temporales cortos, de 3 a 5 días como de media duración, de 6 a 10 días como temporales largos, y de más de 10 días como temporales muy largos. Con la combinación de la intensidad con esta escala de duración obtuvimos 4 grados de peligrosidad estimada de los temporales, clasificación que se puede observar mejor en el CUADRO 2.

Una vez hecha esta clasificación, pasaremos a estudiar los temporales en cada uno de los observatorios intentando averiguar cuales son sus rasgos característicos; y abordaremos también su evolución cronológica a lo largo del período de estudio, con la finalidad fundamental de encontrar tendencias en cuanto a su recurrencia, intensidad y persistencia (en otras palabras, intentar saber si son más o menos frecuentes, intensos y duraderos); pesquisa que, al mismo tiempo, pueda servirnos de indicador de un cambio de comportamiento o tendencia de las condiciones climáticas en Galicia, en particular en el semestre frío del año.

Cuadro 2.

GRADO ESTIMADO DE PELIGROSIDAD DE LOS TEMPORALES DE LLUVIA Y VIENTO Duración

\begin{tabular}{|l|c|c|c|c|}
\hline Intensidad & $\mathbf{1 - 2}$ días & 3-5 días & 6-10 días & >10 días \\
\hline Débil & Grado 1 & Grado 1 & Grado 2 & Grado 3 \\
\hline Moderado & Grado 1 & Grado 2 & Grado 2 & Grado 3 \\
\hline Fuerte & Grado 2 & Grado 2 & Grado 3 & Grado 4 \\
\hline Intenso & Grado 3 & Grado 3 & Grado 4 & Grado 4 \\
\hline
\end{tabular}

Elaboración propia. 


\section{Resultados obtenidos}

A continuación pasaremos a analizar los resultados que hemos conseguido de nuestro análisis de los episodios de temporal de lluvia y viento. Creemos conveniente comenzar por dar una visión sintética de los rasgos más sobresalientes de estos eventos, es decir, las características básicas referidas a las precipitaciones, las rachas máximas de viento y la duración tanto en cuanto a valores medios como extremos haciendo referencia en todo momento a los contrastes espaciales de los mismos. Posteriormente pasaremos ya a exponer el resultado de la clasificación de los episodios de temporal según su intensidad y su grado de peligrosidad, acompañada del estudio de las tendencias seguidas por estos parámetros a lo largo del período de referencia.

El primer hecho destacable es la notable diferencia existente entre el observatorio de A Coruña y los de Lavacolla y Peinador en los resultados obtenidos. De entrada, el número de episodios de temporal localizados en la estación herculina es sensiblemente menor que en las otras dos (CUADRO III): mientras en A Coruña tan sólo hemos conseguido delimi$\operatorname{tar} 45$ episodios, en Lavacolla han sido 193 y en Peinador un total de 170. No quiere decir esto que en A Coruña los temporales sean un hecho aislado y poco frecuente; la explicación hay que buscarla en la menor intensidad de las precipitaciones. Recordemos que una condición inexcusable para que existiese un episodio de temporal de lluvia y viento era que hubiese precipitaciones iguales o superiores a $30 \mathrm{~mm}$ en 24 horas, y en la ciudad herculina solamente hemos podido encontrar en toda la serie 76 días que registrasen tales valores pluviométricos, hecho que constriñe notoriamente la posibilidad de que existan episodios de temporal donde se combinen lluvias intensas con vientos fuertes. En contraste, el viento intenso sí es un meteoro mucho más habitual en A Coruña, con 1104 días que han registrado rachas máximas iguales o superiores a $62 \mathrm{Km} / \mathrm{h}$. El observatorio de Lavacolla, mientras tanto, registró un total de 502 días con precipitaciones iguales o superiores a 30 mm y 1082 días con rachas atemporaladas de viento, lo que nos indica que es un observatorio donde ambos meteoros son de gran importancia y por lo tanto hacen más habitual la presencia de temporales de lluvia y viento (como ya hemos visto). Peinador por su parte anota valores muy parejos de ambas variables, con 522 días de precipitación intensa y 551 de rachas atemporaladas de viento.

Todo esto no es más que un fiel reflejo de las condiciones climáticas habituales de estas comarcas de Galicia: A Coruña, ciudad de emplazamiento eminentemente marítimo, está muy expuesta a los fuertes vientos, pero al mismo tiempo se encuentra bastante bien resguardada de la influencia directa de los vientos de componente SW, que en la comunidad gallega suelen traer las precipitaciones más cuantiosas, de ahí la menor intensidad que en ella alcanzan las precipitaciones. Por su parte, los observatorios de Lavacolla y Peinador están estratégicamente ubicados en los primeros escalones orográficos del W de Galicia, que son los primeros obstáculos que las masas de aire muy cargadas de humedad del SW se encuentran y donde descargan abundantes precipitaciones; a su vez, Lavacolla se encuentra muy al descubierto de esos flujos de viento del SW, en el fondo del pasillo de entrada que le ofrece la Ría de Arousa hasta el interior de la mitad meridional de la provincia de A Coruña, lo que hace que registre fuertes rachas. Esto no sucede en Peinador con tanta frecuencia, pues aunque se ubica muy próximo a la Ría de Vigo está relativamente abrigado de los vientos más intensos por los relieves circundantes (Serra do Galiñeiro sobre todo) que cierran la periferia de la ciudad de Vigo por su flanco S y SW.

Los grandes contrastes en la cuantía e intensidad de las precipitaciones en los episodios de temporal se reflejan con mucha claridad en el CUADRO III. A Coruña queda muy descolgada en las variables que se relacionan con la intensidad y abundancia de las lluvias, 
como la precipitación media diaria máxima o la precipitación acumulada máxima y la acumulada media. En cuanto a las rachas de viento, en cambio, las diferencias son bastante menos importantes; de hecho, se puede apreciar que los valores de la racha máxima absoluta y el valor medio de las rachas máximas son bastante similares. Esto nos lleva a la conclusión de que, mientras en A Coruña los pocos temporales de lluvia y viento registrados se caracterizan por presentar vientos fuertes y lluvias como mucho moderadas, en Lavacolla y Peinador ambos meteoros suelen alcanzar cierta intensidad (en especial en el primero de los casos, donde los fuertes vientos se presentan con más frecuencia que en Peinador), presentándose con cierta frecuencia auténticos temporales de lluvias abundantes y vientos intensos y racheados.

En cuanto a la duración de estos eventos, son A Coruña y Lavacolla los que sufren los episodios más largos, con una media de 5,2 y 4,9 días respectivamente (VER CUADRO 3), mientras que en Peinador la media se queda en 3,8 ; en todo caso, unos valores bastante destacables. Estas diferencias se repiten en los valores máximos, siendo de nuevo A Coruña y Lavacolla los observatorios donde los episodios pueden llegar a durar más días (21 y 20 respectivamente), al tiempo que en Peinador los episodios más largos no pasaron de 13 días de duración. Se puede decir por tanto que los temporales de lluvia y viento no suelen ser eventos fugaces sino que por el contrario persisten un cierto tiempo y en ocasiones pueden prolongarse varios días, a tenor del grado de persistencia e intensidad que alcance la circulación de vientos del $\mathrm{W}$ en nuestras latitudes.

Un último factor que nos queda por aludir es la dirección predominante de las rachas atemporaladas de viento (aquellas que igualan o superan los $62 \mathrm{Km} / \mathrm{h}$ ) durante estos eventos, que también hemos decidido analizar. El resultado, tal como era de esperar, es muy claro: la inmensa mayoría de ellas soplan del $3^{\circ}$ cuadrante (dirección SW), como suele ocurrir en los centros de bajas presiones de la zona templada del Hemisferio Boreal (VER CUADRO 4). En A Coruña suponen el 61.74\% del total (concretamente, 113 de 183), en Lavacolla ascienden al 77.65\% (563 de 725), y en Peinador acaparan el 61.5\% (238 de 387). Sin embargo, hay otras componentes que aún teniendo mucha menos importancia tienen cierta presencia, como la componente $\mathrm{S}$ en el caso de Peinador (65 registros de $180^{\circ}$ ), el $2^{\circ}$ cuadrante en Lavacolla con 104 registros, o el $4^{\circ}$ cuadrante en el propio Peinador y A Coruña con 50 y 32 registros respectivamente, direcciones todas ellas también asociadas al paso de centros depresionarios (los vientos del SW en ocasiones pueden convertirse en vientos del $\mathrm{S}$, y los vientos del NW también pueden soplar con fuerza con advecciones de esta dirección). El $1^{\circ}$ cuadrante apenas tiene relevancia en todos los casos.

Una vez hecha esta semblanza de las características generales de los episodios de temporal y sus contrastes espaciales, pasaremos a analizar la evolución cronológica de tales rasgos. En líneas generales, se puede afirmar que tanto el número de episodios de temporal como su duración experimentaron un descenso, en especial en los últimos 15 ó 20 años de la serie (FIGURAS 2 y 3). En casi todos los parámetros estudiados se puede ver con cierta claridad un contraste entre una primera etapa de valores más elevados, coincidente con las décadas de los años 1960 y 1970, y una segunda etapa caracterizada por presentar unos registros menores y básicamente centrada en las décadas de los 1980 y 1990. Obviamente, y tal como iremos viendo, el contraste entre ambos períodos no es igual de importante en todos los parámetros ni en todos los observatorios; de hecho, de nuevo la ciudad de A Coruña vuelve a diferenciarse por presentar una evolución de sus variables mucho menos contrastada que Lavacolla y Peinador en casi todos los casos. Una vez más, la explicación más plausible hay que buscarla en la ubicación de la ciudad herculina a sotavento de los vientos del SW y su localización más septentrional dentro de Galicia, factores ambos que por un lado, atenúan la influencia de las precipitaciones asociadas a los vientos del $3^{\circ}$ cuadrante y por otro lado, 
Cuadro 3.

ALGUNOS PARÁMETROS SIGNIFICATIVOS DE LOS EPISODIOS DE TEMPORAL DE LLUVIA Y VIENTO

\begin{tabular}{|l|c|c|c|}
\hline & A Coruña & Lavacolla & Peinador \\
\hline Total de episodios & 45 & 193 & 170 \\
\hline Duración media & 5.2 días & 4.9 días & 3.8 días \\
\hline Duración máxima & 21 días (6/26-2-1966) & 20 días (7/26-2-1966) & $\begin{array}{c}13 \text { días (8/20-2-1966; } \\
3 / 15-12-1978 ; \\
11 / 23-12-1981)\end{array}$ \\
\hline $\begin{array}{l}\mathrm{N}^{0} \text { de episodios fuertes } \\
\text { e intensos }\end{array}$ & $4(8.9 \%)$ & $55(28.5 \%)$ & $53(31.2 \%)$ \\
\hline $\begin{array}{l}\mathrm{N}^{0} \text { de episodios de } \\
\text { peligrosidad 3 y 4 }\end{array}$ & $6(13.3 \%)$ & $46(23.8 \%)$ & $23(13.5 \%)$ \\
\hline $\begin{array}{l}\text { Precipitación acumulada } \\
\text { media (total episodios) }\end{array}$ & $80.9 \mathrm{~mm}$ & $143.4 \mathrm{~mm}$ & $126 \mathrm{~mm}$ \\
\hline $\begin{array}{l}\text { Valor medio de las rachas } \\
\text { máximas (total episodios) }\end{array}$ & $79.1 \mathrm{Km} / \mathrm{h}$ & $87.1 \mathrm{Km} / \mathrm{h}$ & $79.3 \mathrm{Km} / \mathrm{h}$ \\
\hline $\begin{array}{l}\text { Precipitación media diaria } \\
\text { más alta }\end{array}$ & $53.4 \mathrm{~mm}(25-10-1990)$ & $127.2 \mathrm{~mm}(13 / 15-10-1987)$ & $97.1 \mathrm{~mm}(25-1-1977)$ \\
\hline $\begin{array}{l}\text { Precipitación media diaria } \\
\text { más baja }\end{array}$ & $7.5 \mathrm{~mm}(13 / 25-3-1963)$ & $8.9 \mathrm{~mm}(7 / 10-3-1981)$ & $14.6 \mathrm{~mm}(21 / 24-3-1981)$ \\
\hline $\begin{array}{l}\text { Precipitación máxima absoluta } \\
\text { en 24 horas }\end{array}$ & $132.7 \mathrm{~mm}(8-3-1999)$ & $218 \mathrm{~mm}(14-10-1987)$ & $175 \mathrm{~mm}(7-12-1978)$ \\
\hline Precipitación acumulada máxima & $188.9 \mathrm{~mm}(6 / 15-12-1978)$ & $580.2 \mathrm{~mm}(31-1 / 14-2-1979)$ & $475.6 \mathrm{~mm}(3 / 15-12-1978)$ \\
\hline $\begin{array}{l}\text { Racha máxima absoluta de viento } \\
\text { (130 Km/h (4-10-1984) }\end{array}$ & $155 \mathrm{Km} / \mathrm{h}(5-2-1972 ;$ & $140 \mathrm{Km} / \mathrm{h}(5-2-1972)$ \\
\hline $\begin{array}{l}\text { Mayor número de episodios de } \\
\text { temporal en un semestre invernal }\end{array}$ & $6(2000-2001)$ & $12(2000-2001)$ & $11(2000-2001)$ \\
\hline
\end{tabular}

Fuente: Datos diarios del INM. Elaboración propia.

Cuadro 4.

$N^{\circ}$ DE DÍAS CON RACHAS ATEMPORALADAS DE VIENTO DURANTE LOS EPISODIOS DE TEMPORAL SEGÚN SU DIRECCIÓN

\begin{tabular}{|l|c|c|c|c|}
\hline Estaciones & $\mathbf{1}^{\mathbf{0}}$ Cuadrante & $\mathbf{2}^{\mathbf{0}}$ Cuadrante & $\mathbf{3}^{\mathbf{0}}$ Cuadrante & $\mathbf{4}^{\mathbf{0}}$ Cuadrantes \\
\hline A Coruña & 5 & 33 & 113 & 32 \\
\hline Lavacolla & 13 & 104 & 563 & 45 \\
\hline Peinador & 2 & 97 & 238 & 50 \\
\hline
\end{tabular}

Fuente: Datos diarios del INM. Elaboración propia. 
ponen al observatorio coruñés más expuesto a influencias de mecanismos pluviométricos del $1^{\circ}$ y del $4^{\circ}$ cuadrante que afectan en mucha menor medida a Lavacolla y Peinador.

Paralelamente a esto, los parámetros relacionados con la intensidad (precipitación acumulada máxima y máxima absoluta, y valor medio de las rachas máximas y racha máxima absoluta) no parecen haber sufrido sustanciales variaciones a lo largo del tiempo. Sí son algo más destacables en cuanto a las precipitaciones acumuladas, pero es un hecho que se puede poner en relación directa con la menor duración de los episodios (al durar menos, acumulan menos precipitación). Un buen ejemplo puede ser la evolución de la precipitación acumulada máxima de los episodios de temporal en Lavacolla y Peinador (FIGURA 4), variable directamente relacionada con la duración de los episodios y que experimenta un sustancial descenso en los años 1980 y en particular en los años 1990.

\subsection{Intensidad y peligrosidad}

A continuación pasamos ya a abordar la última parte del presente artículo, centrada en el análisis de la intensidad y la peligrosidad de los episodios de temporal de lluvia y viento y su evolución cronológica. A priori, ambas variables necesariamente irán muy de la mano de la intensidad alcanzada por las precipitaciones y el viento, de forma que presumiblemente volverán a repetirse los contrastes espaciales ya aludidos y, en cierto modo, la evolución cronológica igualmente tenderá a repetirse. El análisis confirma en casi todos sus extremos estas premisas. Así, A Coruña sufre los episodios menos intensos y peligrosos: como podemos ver en el CUADRO 3; tan sólo 4 de los 45 episodios de temporal acotados en este observatorio se pueden considerar fuertes, lo que supone apenas un $8,88 \%$ del total (ninguno de ellos llega a ser intenso), mientras sólo 6 llegan a categoría 3 de peligrosidad (el $13,33 \%$ ) y de ellos tampoco hay ninguno que alcance el grado 4. En contraste, Lavacolla y Peinador anotan cifras muy superiores. En el caso de Peinador, son fuertes o intensos 53 episodios (un $31,18 \%$ del total) y de estos se consideran un total de 23 como grado 3 de peligrosidad (el 13,53\%, de los cuales ninguno llega a grado 4); en Lavacolla, mientras tanto, alcanzan a ser fuertes o intensos 55 episodios (un 28,5\%) de los que 46 (un $23,83 \%$ ) alcanzan el grado 3 de peligrosidad, y de estos existen 4 que alcanzan el grado de peligrosidad 4, siendo el único observatorio donde los hay. Todo esto, en síntesis, nos dice que A Coruña soporta temporales poco intensos y peligrosos, fundamentalmente debido a que en esta ciudad las precipitaciones no suelen ser muy fuertes, mientras que Peinador y Lavacolla sufren con cierta frecuencia temporales que alcanzan importante intensidad y peligrosidad, sobre todo en el caso de Lavacolla, único observatorio de los estudiados donde de vez en cuando se puede registrar un temporal de máximo grado de peligrosidad.

En cuanto a la evolución cronológica de estas variables, un primer análisis de los datos parece dar a ver una cierta diferencia entre ambas variables: mientras por un lado el grado de peligrosidad de los temporales experimenta un retroceso en los últimos 15 ó 20 años, en concordancia con lo que ya se ha visto para la mayoría de los parámetros de los temporales, la intensidad de los mismos apenas parece haber sufrido cambios sustanciales. De hecho, en lo que respecta a la intensidad, tan sólo se nota un cierto descenso circunscrito a los años 1990, pues en los años 1980 lo que se nota es el descenso en el número de episodios pero no de la intensidad de los mismos en sí. No sucede lo mismo para la peligrosidad, donde sí es evidente un retroceso, siendo Lavacolla un caso paradigmático: en los años 1960 y 1970 registra 33 episodios de grados 3 y 4, a la par que en los años 1980 y 1990 esta cifra se reduce a tan sólo 15 . No obstante, y para contrastar mejor estos hechos, hemos decidido emplear un índice de intensidad y de peligrosidad invernal de los temporales y conocer su evolución cronológica. El citado índice lo elaboramos del siguiente modo: se toman todos 

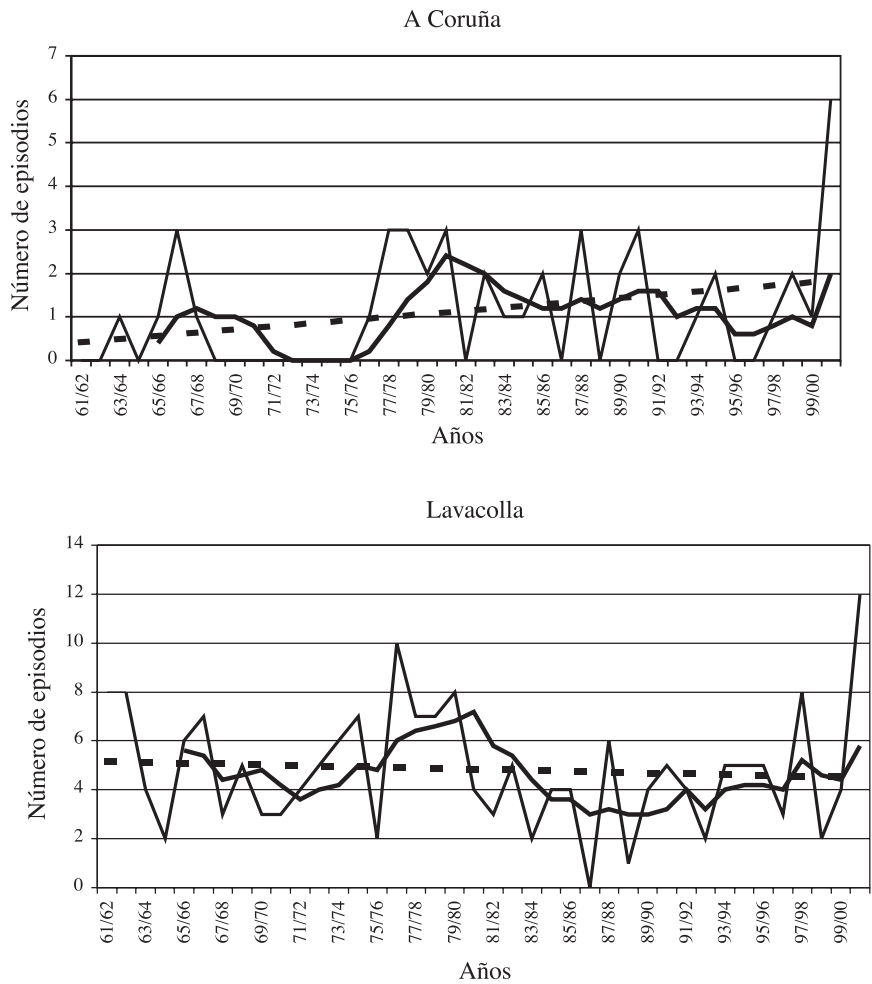

Peinador

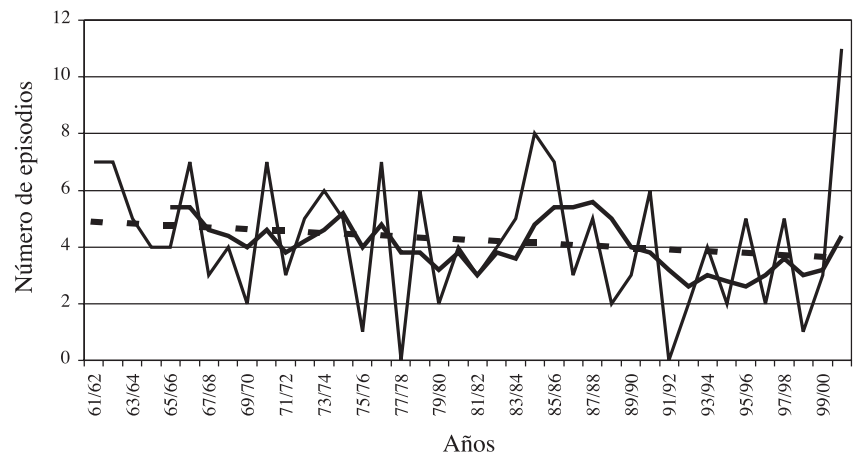

Número de episodios

Media móvil (5 años)

Recta de tendencia

FiguRA 2. Evolución del número de episodios de temporal. 


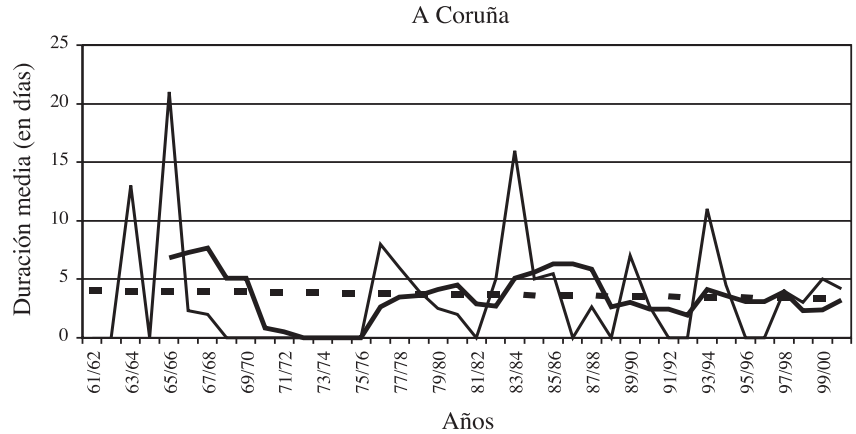

Lavacolla
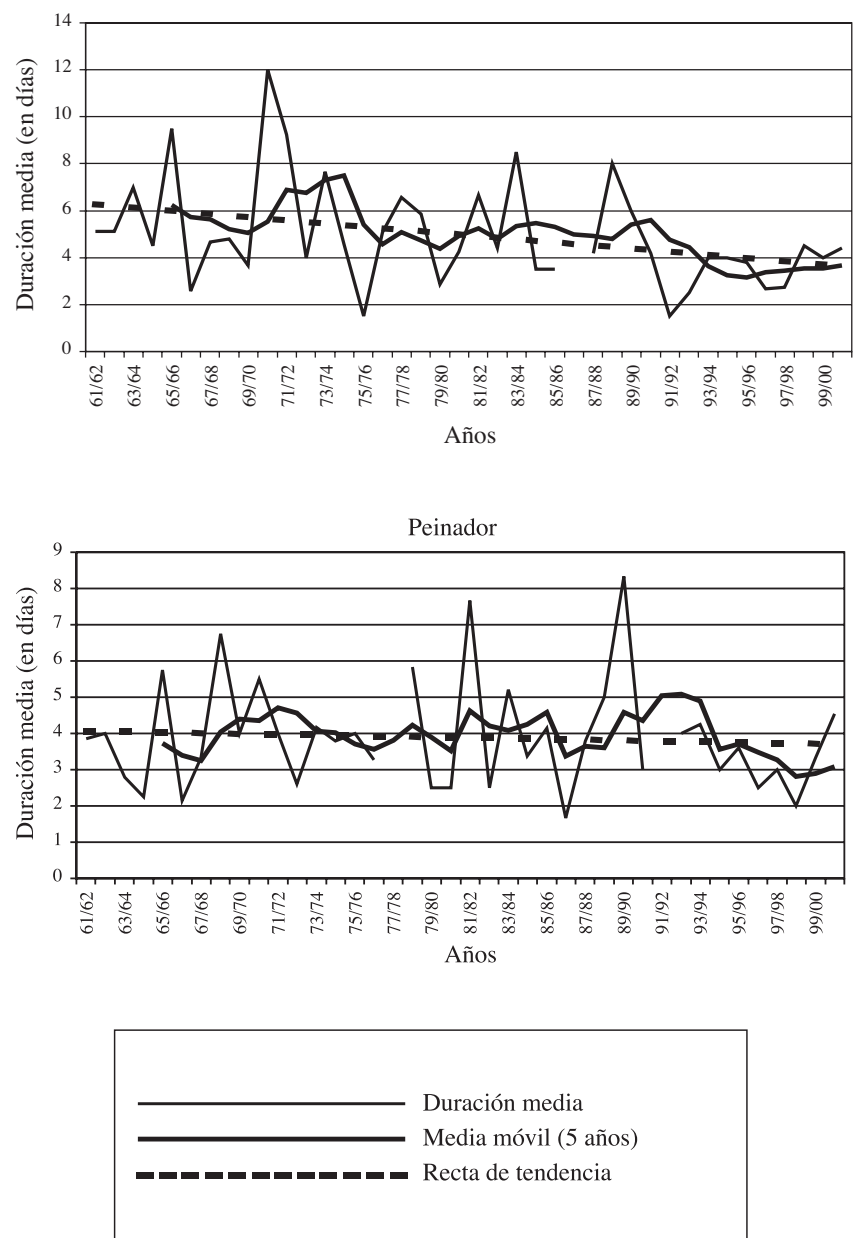

FIGURA 3. Evolución de la duración media de los episodios de temporal. 
Lavacolla

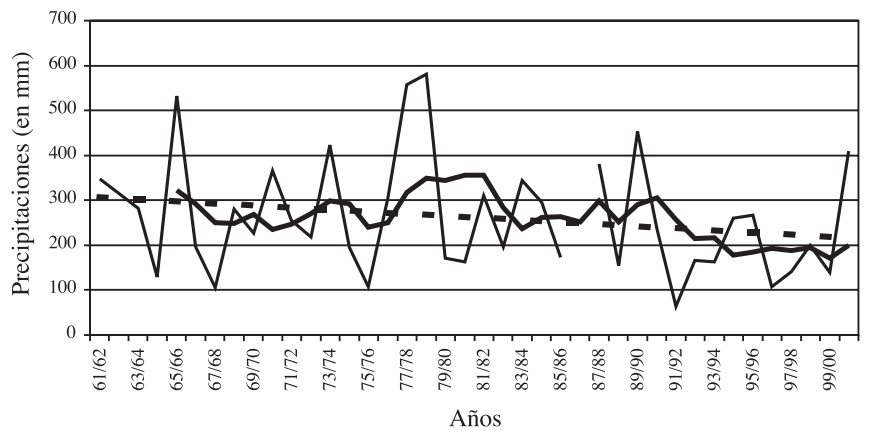

Peinador

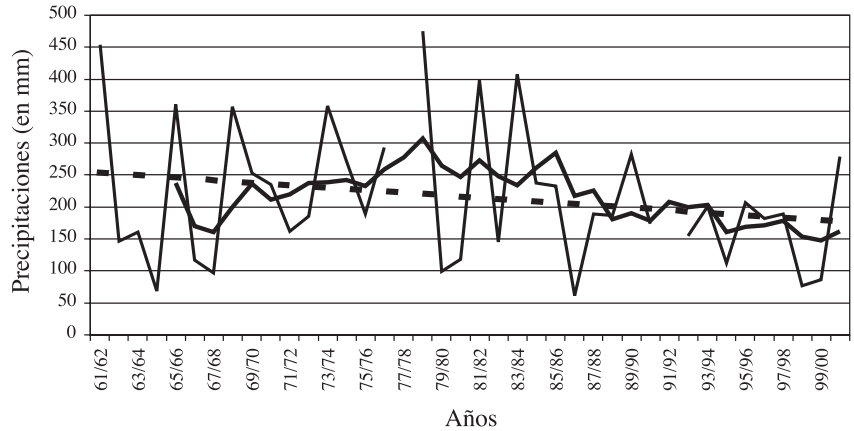

Precipitación acum. máx.

Media móvil (5 años)

Recta de tendencia

FIGURA 4. Evolución de la precipitación acumulada máxima. 
A Coruña

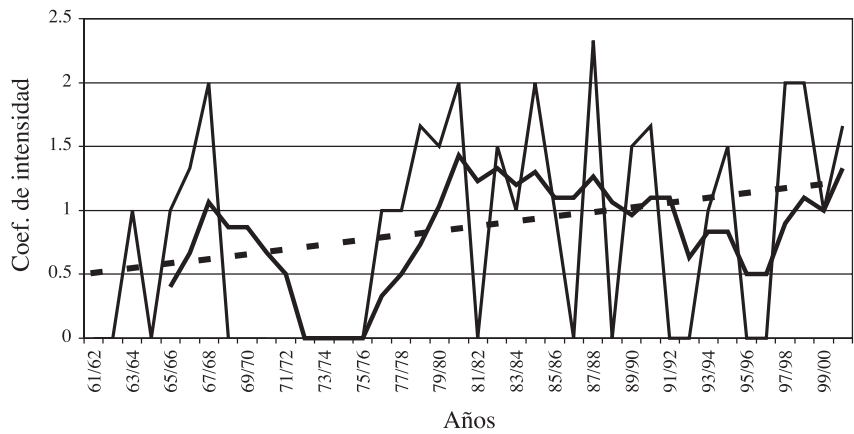

Lavacolla

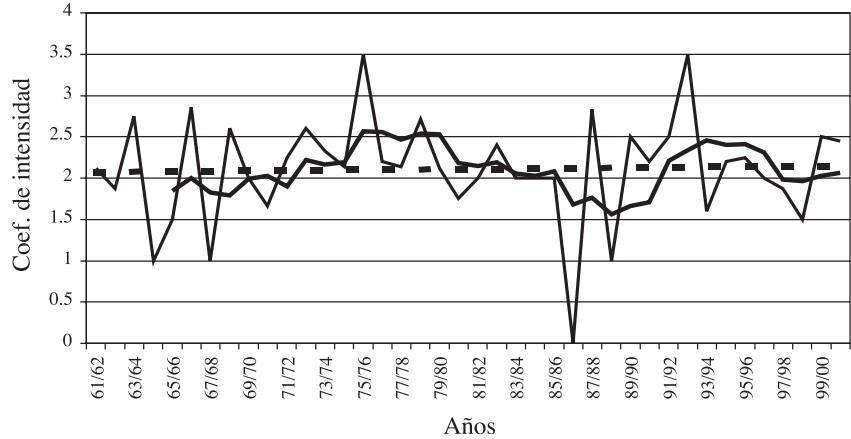

Peinador

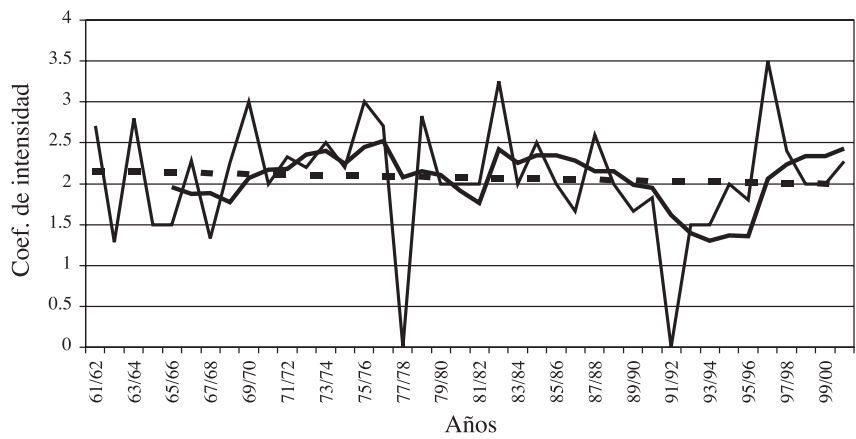

Coeficiente de intensidad

Media móvil (5 años)

Recta de tendencia

FiguRA 5. Evolución del coeficiente de intensidad de los episodios de temporal. 


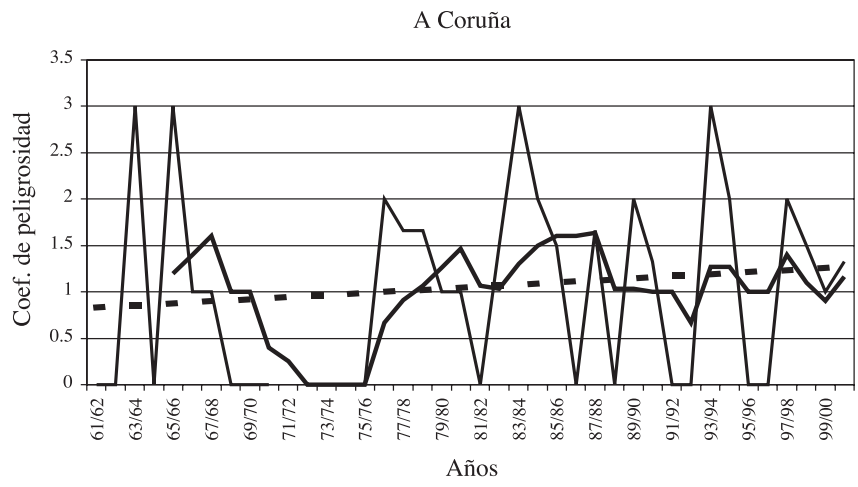

Lavacolla

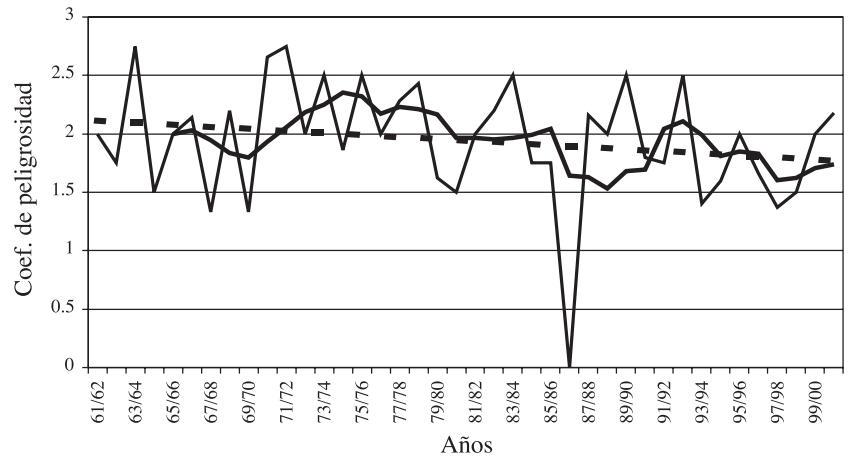

Peinador
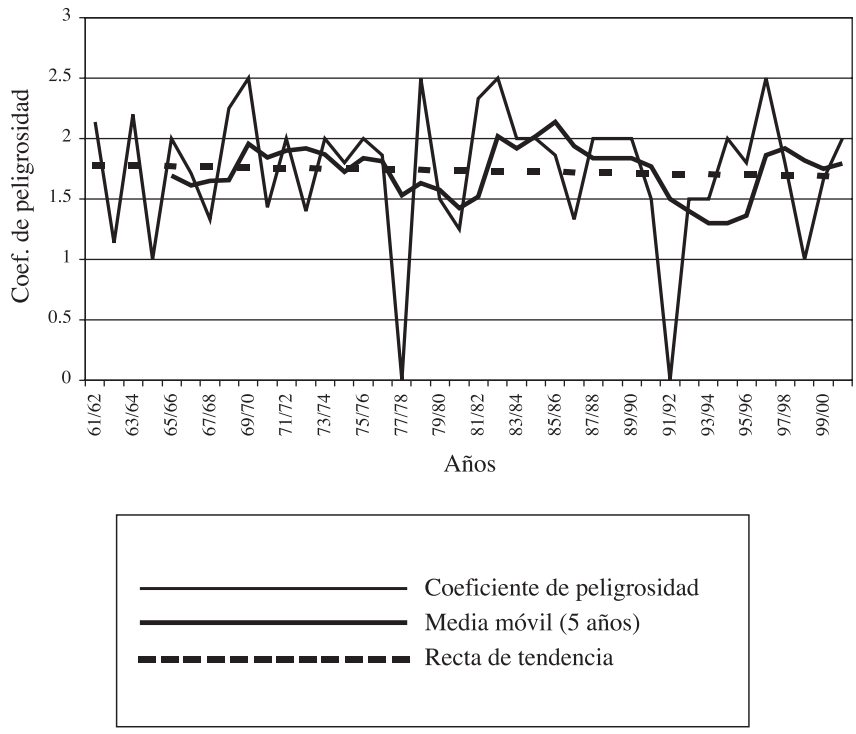

FIGURA 6. Evolución del coeficiente de peligrosidad de los episodios de temporal. 
los episodios de temporal localizados en el observatorio durante un semestre invernal y a cada uno de ellos se le adjudica un valor según su intensidad y su grado de peligrosidad (valor 1 para los episodios débiles y los de grado 1 de peligrosidad, valor 2 para los episodios moderados y de grado de peligrosidad 2, etc), esos valores se suman y el resultado se divide por el número de episodios de ese semestre invernal; el cociente resultante es el índice de peligrosidad y de intensidad que buscamos. Con él, elaboramos las correspondientes gráficas evolutivas de ambos parámetros para los 3 observatorios (FIGURAS 5 y 6), que complementan así los referidos al número de episodios por intensidad y peligrosidad. Las primeras impresiones se confirman, al menos en parte. Mientras que, por norma general, apenas hay cambios en cuanto a la intensidad, con unas rectas de ajuste que apenas se apartan de la horizontal, en cuanto a la peligrosidad ya nos encontramos con casos donde sí se nota un retroceso; el mejor ejemplo es Lavacolla, donde los últimos 15 años asisten a un claro descenso en la peligrosidad de los temporales. Así pues, los temporales de lluvia y viento, si bien son menos numerosos y más cortos, son casi igual de intensos y tienden a ser menos peligrosos por el hecho de ser menos duraderos.

\section{Conclusiones}

Como colofón a lo que acabamos de estudiar, podemos sintetizar los resultados en las siguientes conclusiones generales:

1) Los episodios de temporal de lluvia y viento en Galicia son un evento climático de gran importancia, tanto en cuanto a su intensidad como en cuanto a su frecuencia, constituyéndose como un riesgo climático de primera magnitud, en especial en el litoral y cercanías. Paradójicamente, están poco estudiados en la climatología gallega y española.

2) Los contrastes territoriales en cuanto a las características de los episodios de temporal son notables. Mientras lugares como A Coruña sufren temporales donde el viento sopla con fuerza y las lluvias, sin embargo, no son muy intensas, otros como Peinador y sobre todo Lavacolla sí llegan a presentar temporales de lluvias intensas y vientos muy violentos con cierta frecuencia. La disposición de las formas del relieve y la mayor o menor exposición a los vientos de componente $\mathrm{SW}$ tiene mucho que ver en ello.

3) Parece constatarse una tendencia a reducirse el número, la duración y la peligrosidad potencial de los episodios de temporal, en especial en los últimos 20 años. En cambio, su intensidad no parece presentar cambios demasiado significativos.

\section{Bibliografía}

CASTILLO RODRÍGUEZ, F. (2001): «Estudio sinóptico y analítico de la precipitación en Galicia». Tesis doctoral (inédita). Universidade de Santiago de Compostela.

CASTILLO RODRÍGUEZ, F; PÉREZ ALBERTI, A. (1993): «Tipos de tiempo lluviosos durante el otoño-invierno en el litoral noroccidental gallego». Notes de Geografía Físi$c a \mathrm{n}^{\circ}$ 22, pp. 99-112. Universidad de Barcelona.

MARTÍ EZPELETA, A. (2002): «Evolución secular das precipitacións de inverno en Galicia». Revista Semata $\mathrm{N}^{\circ} 1$. Universidade de Santiago de Compostela.

MARTÍ EZPELETA, A.; GARCÍA MARTÍNEZ, E.; MIRAGAYA VERAS, A. (1998): «Rachas máximas y temporales de viento en Galicia». Lurralde n ${ }^{\circ} 21$, pp. 261-280. Instituto vasco de geografia. 
MARTÍ EZPELETA, A.; PÉREZ SÁNCHEZ, J.A. (1997): «El impacto de los temporales sobre el sector pesquero gallego: el puerto de Laxe». En «Dinámica litoral-interior» vol. II. Universidade de Santiago de Compostela. pp. 725-736.

OLCINA CANTOS, J. (1994): «Riesgos climáticos en la Península Ibérica». Editorial Penthalón. Madrid. 440 pp.

RASILLA ÁlVAREZ, D.; GARCÍA CODRÓN, J.C.; GARMENDIA PEDRAJA, C. (2002): «Los temporales de viento: propuesta metodológica para el análisis de un fenómeno infravalorado». En CUADRAT, J.M.; VICENTE, S.M.; SAZ, M.A. (eds.): «La información climática como herramienta de gestión ambiental»; VII Reunión Nacional de Climatología, Albarracín (Teruel). Universidad de Zaragoza. pp. 129-136. 